\title{
La Ley de dependencia: costes reales y financiación total durante el periodo 2012-2015
}

\section{The dependency law: real costs and funding through the period 2012-2015}

\section{Palabras clave}

Ley de dependencia, coste, gasto público, servicios, prestaciones económicas, financiación finalista, financiación adicional.

\section{Keywords}

Dependency law, costs, social expenditure, economic benefits, finalist funding, additional funding.

\section{Introducción}

El objetivo de este artículo es analizar la financiación del Sistema para la Autonomía y Atención a la Dependencia (en adelante SAAD) durante los años 20I 2 a 2015 . En un artículo anterior hemos expuesto ya la evolución de la financiación de la Ley de dependencia entre los años 20I 2 y 2014 (Beitia, 20I5). En el presente estudio se amplía y actualiza aquel trabajo incluyendo el análisis del ejercicio correspondiente a 2015 , puesto que este periodo muestra unos datos homogéneos, lo que nos facilitará la realización de comparaciones fiables.

Para conocer la situación del SAAD durante esos años, basta con comprobar los datos oficiales comunicados por las comunidades autónomas al Instituto de Mayores y Servicios Sociales, Imserso, y publicados por éste.

Así, el número de beneficiarios con prestación, es decir, las personas realmente atendidas: se puede apreciar en el Gráfico I que durante los años 2012 a 2015 las cifras de beneficiarios con prestación se han mantenido en torno a las 750.000 personas. En el año 2015 y como consecuencia de la incorporación de personas con

\section{Rafael Beitia}

<rbeitia@imserso.es>

Adjunto a la directora general del Instituto de Mayores y Servicios Sociales (Imserso). Ministerio de Sanidad, Servicios Sociales e Igualdad.

\section{Balbino Pardavila}

<bpardavila@imserso.es>

Jefe de servicio en el gabinete de la directora general del Instituto de Mayores y Servicios Sociales (Imserso). Ministerio de Sanidad, Servicios Sociales e Igualdad

Para citar:

Beitia, R. y Pardavila, B. (20I6): "La Ley de dependencia: costes reales y financiación total durante el periodo 20I 2-20I 5". Revista Española de Discapacidad, 4 (2): I89-204.

Doi: <https://doi.org/IO.5569/23405104.04.02.IO> 
Gráfico 1. Personas beneficiarias de prestación del Sistema para la Autonomía y Atención a la
Dependencia (2008-2015)

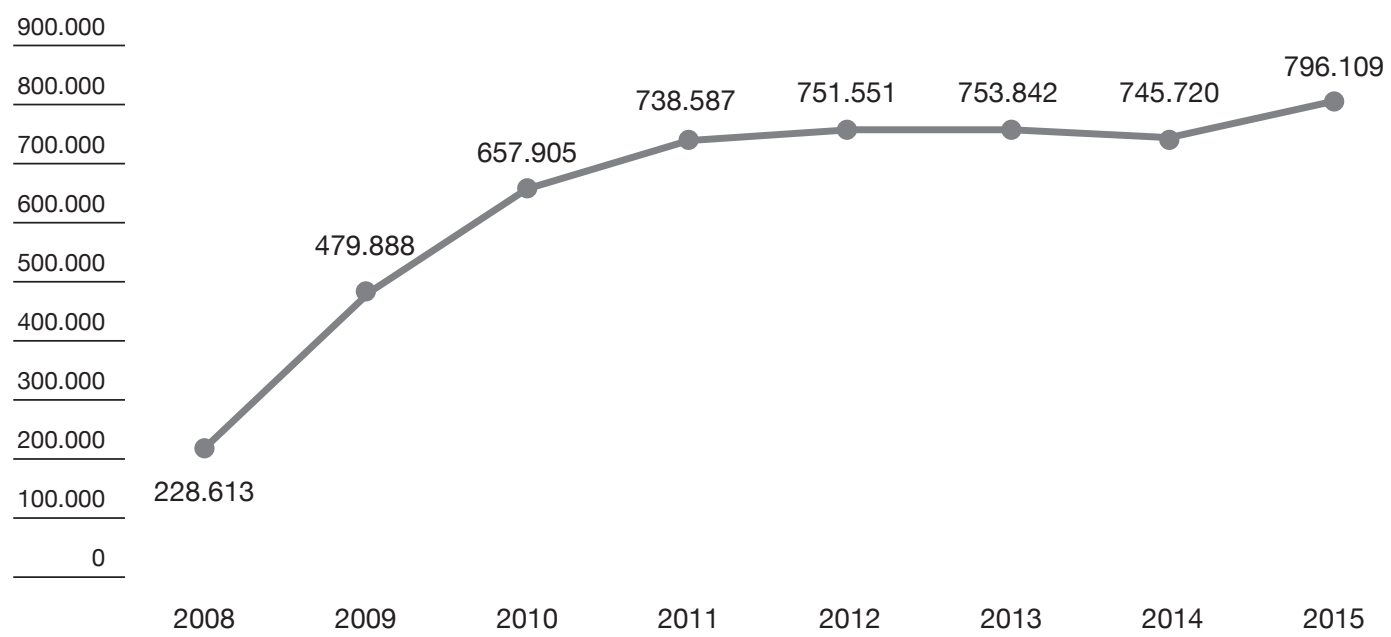

Fuente: Sistema de Información del Sistema para la Autonomía y Atención a la Dependencia (SISAAD). Datos regularizados de acuerdo con los Informes 977/20I3 y I.035/20I4 del Tribunal de Cuentas.

Grado I de dependencia, los beneficiarios con prestación han aumentado hasta los 796.I09, lo que evidenciaría cuán lejos se encuentra el SAAD, no ya de una pretendida "caída libre" (Montserrat, 20I3) sino, ni siquiera, de una supuesta "demolición controlada" (AEDGSS, 20I2), calificativos muy extendidos, quizá por mediáticos, pero alejados de la realidad.

En definitiva, la verdadera situación del número de personas dependientes atendidas por el SAAD durante los años 2012 a 2015 , atendiendo a valores absolutos, se podría definir como de estabilidad e, incluso, de crecimiento en el último año.

El objeto de este artículo es poder fijar el coste de esas 75 I.5 5I, 753.842, 745.720 y 796.106 personas atendidas por el SAAD en los años 2OI2, 2OI3, 20I4 y $20 \mathrm{I} 5$ respectivamente, y exponer cómo se ha financiado.

\section{El coste del Sistema para la Autonomía y Atención a la Dependencia}

Existe una relación directa entre la financiación y los costes de la atención a la dependencia, por lo que para conocer aquélla se hace necesario en primer lugar estimar éstos ${ }^{\mathrm{I}}$.

\subsection{Metodología de cálculo del coste de la atención a la dependencia}

Para determinar el coste de la atención a la dependencia en los años 2012 a 2015 partimos de las prestaciones ${ }^{2}$, tanto de servicios como de prestaciones económicas, reconocidas por las comunidades autónomas.

\footnotetext{
I. El Tribunal de Cuentas (20I4) señala en su informe sobre las medidas de gestión y control adoptadas por las comunidades autónomas para la adecuada aplicación de la Ley de dependencia, que no resulta nada sencillo poder estimar el coste que supone la atención a la dependencia en cada año dada la falta de datos relativos a las aportaciones realizadas, tanto por las comunidades autónomas como por las propias personas beneficiarias. 2. Con el término genérico 'prestaciones' nos estamos refiriendo aquí tanto a los servicios del catálogo de la Ley de dependencia como a las prestaciones económicas.
} 
Se han contemplado los servicios ${ }^{3}$ clasificándolos en distintos escenarios, según la categoría del correspondiente servicio, el grado y nivel de dependencia, el nivel de renta y la comunidad autónoma de residencia.

En cuanto a las prestaciones económicas ${ }^{4}$, se han diseñado igualmente diferentes escenarios en función del tipo de prestación, del grado y nivel de dependencia y del nivel de renta de la persona.

Se ha desarrollado un modelo de estimación que distingue, por un lado, servicios y, por otro, prestaciones económicas, asignando a ambos un indicador de referencia: un coste medio a los diferentes servicios y una cuantía media a las diferentes prestaciones económicas.

Relacionando ambos factores, es decir, multiplicando el número de prestaciones por el indicador de referencia, según corresponda, se obtiene la cifra del coste anual de atención del SAAD.

3. El artículo I 5 de la Ley de dependencia recoge las tipologías de servicios que se han tenido en cuenta para la realización de este estudio:

- Servicio de teleasistencia.

- Servicio de ayuda a domicilio.

- Servicios de centro de día y noche.

- Servicios de atención residencial.

El servicio de Prevención de las Situaciones de Dependencia y Promoción de la Autonomía Personal (PAPD) se ha excluido de este estudio.

4. Prestación económica vinculada al servicio, prestación económica de cuidados en el entorno familiar y apoyo a cuidadores no profesionales, así como prestación económica para cuidador personal (art. I 5 Ley de dependencia).

\section{a. El coste de los servicios}

El proceso de cálculo para la obtención del coste medio de los servicios ha considerado tres fuentes de información:

I. El informe Los Servicios Sociales destinados a las personas mayores en España a 3 I de diciembre de 20I2, publicado por el Imserso (2015) y que muestra los precios públicos para cada uno de los cuatro servicios.

2. Fuentes del sector de servicios sociales: datos solicitados por el Imserso a empresas del sector.

3. El estudio Una aproximación al coste de la dependencia en España y su financiación de los profesores de la Universidad de Valladolid Dolores Prada y Luis Borge (20I 5 ).

Se han homogeneizado los datos procedentes de las tres fuentes de información, de tal manera que respondan a un esquema de un indicador por servicio expresado en euros al mes o a la hora, según corresponda, como se muestra en la Tabla I. La media de estos datos es el indicador de coste que se ha utilizado para la estimación del coste medio de los servicios.

\begin{tabular}{|c|c|c|c|c|}
\hline Fuentes & $\begin{array}{l}\text { Teleasistencia } \\
\text { (€/mes) }\end{array}$ & $\begin{array}{c}\text { Ayuda a } \\
\text { domicilio } \\
\text { ( } € / \text { hora })\end{array}$ & $\begin{array}{l}\text { Centros de } \\
\text { día/noche } \\
\text { (€/mes) }\end{array}$ & $\begin{array}{l}\text { Atención } \\
\text { residencial } \\
\text { (€/mes) }\end{array}$ \\
\hline Informe Imserso 2012 & 23,94 & 14,01 & 861,48 & $1.658,13$ \\
\hline Fuentes del sector & 20,00 & 17,00 & $1.063,52$ & $2.542,54$ \\
\hline $\begin{array}{l}\text { Prada y Borge } \\
\text { (U. Valladolid) }\end{array}$ & 20,00 & 15,00 & 873,06 & $1.774,05$ \\
\hline Indicador de coste & 21,31 & 15,34 & 932,69 & $1.991,57$ \\
\hline
\end{tabular}

Fuente: Imserso. Elaboración propia. 


\section{b. La cuantía de las prestaciones económicas}

La cuantía media de las prestaciones económicas se ha calculado partiendo de las cuantías máximas establecidas en la disposición transitoria décima del Real Decreto-ley 20/201 2, de $\mathrm{I} 3$ de julio, de medidas para garantizar la estabilidad presupuestaria y de fomento de la competitividad, con base en la tipología de prestación y el grado y nivel reconocido. Además, se han aplicado las fórmulas relativas a la aportación de la persona beneficiaria recogidas en el Acuerdo de Mejora del SAAD, publicado en el BOE por Resolución de I 3 de julio de 20I2, para determinar la cuantía concedida concreta de cada prestación. En todo caso, se ha tratado de ponderar el mayor número de factores posibles, en orden a calcular una cuantía media que se ajuste lo máximo posible a la realidad social y económica de las personas beneficiarias.

\begin{tabular}{l}
\hline Tabla 2. Cuantía media de las prestaciones \\
económicas \\
\begin{tabular}{|l|c|c|c|}
\hline & $\begin{array}{c}\text { Prestación } \\
\text { económica } \\
\text { vinculada } \\
\text { al servicio }\end{array}$ & $\begin{array}{c}\text { Prestación } \\
\text { económica } \\
\text { para } \\
\text { cuidados } \\
\text { en el } \\
\text { entorno } \\
\text { familiar }\end{array}$ & $\begin{array}{c}\text { Prestación } \\
\text { económica } \\
\text { para } \\
\text { asistencia } \\
\text { personal }\end{array}$ \\
\hline $\begin{array}{l}\text { Cuantía } \\
\text { media }\end{array}$ & $\begin{array}{c}508,77 \\
€ / \text { mes }\end{array}$ & $\begin{array}{c}319,64 \\
€ / \text { mes }\end{array}$ & $\begin{array}{c}494,57 \\
€ / \text { mes }\end{array}$ \\
\hline
\end{tabular}
\end{tabular}

Fuente: Imserso y SISAAD.

\section{c. Coste de la Ley de dependencia}

El método de estimación del coste total de la Ley de dependencia se basa en la multiplicación del número de prestaciones por su coste.

Para ello se ha desarrollado un modelo de estimación que contempla las 934.3 I 6 prestaciones de 20I2, las 9I 6.767 correspondientes a $20 \mathrm{I}_{3}$, las 898.332 de $20 \mathrm{I} 4$ y las 916.602 de 2015. A continuación, estas prestaciones se han puesto en relación con los indicadores de coste respectivos, esto es, con los costes medios de cada servicio y con las cuantías medias reconocidas de cada prestación económica, de manera que se pudiera llevar a cabo la multiplicación de cada servicio y prestación por su indicador.

Realizadas las operaciones expuestas anteriormente se obtiene el resultado del coste del SAAD, para cada año:

- 2012: 6.895 millones de euros.

- 2013: 6.858 millones de euros

- 2014: 7.090 millones de euros.

- 20I5: 7.449 millones de euros.

Esta misma operación se puede realizar por cada una de las comunidades autónomas y en cada uno de los años de 2012 a 2015 . Los resultados que obtenemos son los que se muestran en la Tabla 3, en la que podemos observar el mayor peso porcentual de Andalucía, Cataluña y la Comunidad de Madrid, como lógico trasunto de su mayor población absoluta, seguidas a cierta distancia por Castilla y León, Galicia y País Vasco, en las que el factor población de mayor edad presenta un gran peso.

Bastante coincidentes con esta estimación de costes son tanto el Tribunal de Cuentas (20I4), que lo cifra para el año 20 I 2 en 7.084 millones de euros con información facilitada por las comunidades autónomas, como el estudio de Prada y Borge (2015), que lo calcula en 6.509 millones de euros en el año 2013.

\section{Financiación del SAAD}

Una vez determinado el coste de la atención a la dependencia, se analiza a continuación la financiación y las aportaciones realizadas por las administraciones públicas, Administración General del Estado y comunidades autónomas, así como las personas beneficiarias, de acuerdo con la legislación vigente aplicable al respecto. 
Tabla 3. Coste del SAAD en 2012-2015 por comunidades autónomas

\begin{tabular}{|l|l|c|}
\hline \multicolumn{2}{ll}{2012} & $\begin{array}{c}\text { \% sí } \\
\text { total }\end{array}$ \\
\hline ANDALUCIA & $\mathbf{1 . 6 1 2 . 8 2 5 . 5 6 7}$ & $23,4 \%$ \\
\hline ARAGON & $\mathbf{1 6 5 . 6 8 1 . 8 6 1}$ & $2,4 \%$ \\
\hline ASTURIAS & $\mathbf{1 4 0 . 4 6 4 . 1 7 1}$ & $2,0 \%$ \\
\hline ISLAS BALEARES & $\mathbf{8 8 . 3 0 8 . 7 6 8}$ & $1,3 \%$ \\
\hline CANARIAS & $\mathbf{1 1 6 . 5 6 2 . 2 7 1}$ & $1,7 \%$ \\
\hline CANTABRIA & $\mathbf{1 2 7 . 5 4 7 . 6 3 7}$ & $1,8 \%$ \\
\hline CASTILLA Y LEON & $\mathbf{5 5 8 . 5 6 2 . 6 9 6}$ & $8,1 \%$ \\
\hline C. LA MANCHA & $\mathbf{3 9 0 . 2 8 3 . 4 0 6}$ & $5,7 \%$ \\
\hline CATALUÑA & $\mathbf{1 . 0 5 3 . 0 9 9 . 0 6 0}$ & $15,3 \%$ \\
\hline C. VALENCIANA & $\mathbf{4 6 0 . 9 5 0 . 2 2 9}$ & $6,7 \%$ \\
\hline EXTREMADURA & $\mathbf{1 7 1 . 6 2 6 . 0 7 9}$ & $2,5 \%$ \\
\hline GALICIA & $\mathbf{4 0 8 . 6 2 3 . 0 1 7}$ & $5,9 \%$ \\
\hline C. de MADRID & $\mathbf{8 9 2 . 2 8 1 . 5 0 3}$ & $12,9 \%$ \\
\hline MURCIA & $\mathbf{1 8 5 . 0 8 9 . 8 7 3}$ & $2,7 \%$ \\
\hline LA RIOJA & $\mathbf{8 1 . 0 1 1 . 9 0 9}$ & $1,2 \%$ \\
\hline NAVARRA & $\mathbf{6 7 . 5 7 5 . 5 9 9}$ & $1,0 \%$ \\
\hline PAÍS VASCO & $\mathbf{3 7 4 . 7 7 0 . 2 8 5}$ & $5,4 \%$ \\
\hline TOTAL & $\mathbf{6 . 8 9 5 . 2 6 3 . 9 3 2}$ & $\mathbf{1 0 0 , 0 \%}$ \\
\hline
\end{tabular}

\begin{tabular}{|l|l|c|}
\hline \multicolumn{2}{|l|}{2013} & $\begin{array}{c}\% \text { sí } \\
\text { total }\end{array}$ \\
\hline ANDALUCIA & $\mathbf{1 . 4 7 7 . 2 0 6 . 1 4 0}$ & $21,5 \%$ \\
\hline ARAGON & $\mathbf{1 4 9 . 4 2 0 . 1 9 2}$ & $2,2 \%$ \\
\hline ASTURIAS & $\mathbf{1 3 8 . 0 6 1 . 4 9 9}$ & $2,0 \%$ \\
\hline ISLAS BALEARES & $\mathbf{8 4 . 8 9 3 . 6 3 7}$ & $1,2 \%$ \\
\hline CANARIAS & $\mathbf{1 1 9 . 8 6 6 . 8 0 8}$ & $1,7 \%$ \\
\hline CANTABRIA & $\mathbf{1 4 3 . 9 8 0 . 2 4 3}$ & $2,1 \%$ \\
\hline CASTILLA Y LEON & $\mathbf{5 4 9 . 7 1 3 . 5 4 0}$ & $8,0 \%$ \\
\hline C. LA MANCHA & $\mathbf{4 0 0 . 7 7 0 . 0 5 6}$ & $5,8 \%$ \\
\hline CATALUÑA & $\mathbf{1 . 1 4 2 . 1 6 6 . 9 1 4}$ & $16,7 \%$ \\
\hline C. VALENCIANA & $\mathbf{4 2 5 . 7 5 4 . 2 5 0}$ & $6,2 \%$ \\
\hline EXTREMADURA & $\mathbf{1 8 2 . 7 3 6 . 1 2 6}$ & $2,7 \%$ \\
\hline GALICIA & $\mathbf{4 3 9 . 2 7 5 . 5 1 5}$ & $6,4 \%$ \\
\hline C. de MADRID & $\mathbf{8 7 1 . 2 6 1 . 2 0 3}$ & $12,7 \%$ \\
\hline MURCIA & $\mathbf{1 8 1 . 1 2 0 . 7 8 1}$ & $2,6 \%$ \\
\hline LA RIOJA & $\mathbf{7 8 . 0 8 2 . 6 7 4}$ & $1,1 \%$ \\
\hline NAVARRA & $\mathbf{7 0 . 1 1 2 . 5 3 0}$ & $1,0 \%$ \\
\hline PAÍS VASCO & $\mathbf{4 0 3 . 2 4 5 . 0 9 7}$ & $5,9 \%$ \\
\hline TOTAL & $\mathbf{6 . 8 5 7 . 6 6 7 . 2 0 6}$ & $\mathbf{1 0 0 , 0 \%}$ \\
\hline
\end{tabular}

\begin{tabular}{|l|l|c|}
\hline \multicolumn{2}{ll}{2014} & $\begin{array}{c}\text { \% sí } \\
\text { total }\end{array}$ \\
\hline ANDALUCIA & $\mathbf{1 . 3 9 3 . 3 1 6 . 7 8 3}$ & $19,7 \%$ \\
\hline ARAGON & $\mathbf{1 4 9 . 8 8 4 . 3 3 3}$ & $2,1 \%$ \\
\hline ASTURIAS & $\mathbf{1 3 6 . 8 3 2 . 6 2 3}$ & $1,9 \%$ \\
\hline ISLAS BALEARES & $\mathbf{9 2 . 4 2 6 . 6 6 1}$ & $1,3 \%$ \\
\hline CANARIAS & $\mathbf{1 2 6 . 8 3 0 . 2 3 7}$ & $1,8 \%$ \\
\hline CANTABRIA & $\mathbf{1 5 1 . 7 2 7 . 1 3 4}$ & $2,1 \%$ \\
\hline CASTILLA Y LEON & $\mathbf{5 4 8 . 5 6 4 . 1 1 4}$ & $7,7 \%$ \\
\hline C. LA MANCHA & $\mathbf{4 0 8 . 8 5 8 . 8 1 9}$ & $5,8 \%$ \\
\hline CATALUÑA & $\mathbf{1 . 3 3 2 . 7 4 0 . 0 5 5}$ & $18,8 \%$ \\
\hline C. VALENCIANA & $\mathbf{4 0 3 . 7 6 6 . 8 1 9}$ & $5,7 \%$ \\
\hline EXTREMADURA & $\mathbf{1 9 9 . 7 2 4 . 0 5 6}$ & $2,8 \%$ \\
\hline GALICIA & $\mathbf{4 2 9 . 1 8 2 . 5 5 9}$ & $6,1 \%$ \\
\hline C. de MADRID & $\mathbf{9 1 2 . 9 8 4 . 1 2 9}$ & $12,9 \%$ \\
\hline MURCIA & $\mathbf{2 0 8 . 0 9 9 . 7 2 6}$ & $2,9 \%$ \\
\hline LA RIOJA & $\mathbf{7 5 . 2 4 9 . 6 9 7}$ & $1,1 \%$ \\
\hline NAVARRA & $\mathbf{7 2 . 6 9 3 . 0 5 5}$ & $1,0 \%$ \\
\hline PAÍS VASCO & $\mathbf{4 4 6 . 8 5 6 . 1 2 5}$ & $6,3 \%$ \\
\hline TOTAL & $\mathbf{7 . 0 8 9 . 7 3 6 . 9 2 6}$ & $\mathbf{1 0 0 , 0 \%}$ \\
\hline
\end{tabular}

\begin{tabular}{|l|l|c|}
\hline \multicolumn{2}{|l}{2015} & $\begin{array}{c}\% \\
\text { total }\end{array}$ \\
\hline ANDALUCIA & $\mathbf{1 . 4 8 9 . 9 5 5 . 8 7 1}$ & $20,0 \%$ \\
\hline ARAGON & $\mathbf{1 5 1 . 5 1 7 . 6 0 8}$ & $2,0 \%$ \\
\hline ASTURIAS & $\mathbf{1 3 7 . 8 5 0 . 1 7 4}$ & $1,9 \%$ \\
\hline ISLAS BALEARES & $\mathbf{9 4 . 7 0 9 . 1 3 7}$ & $1,3 \%$ \\
\hline CANARIAS & $\mathbf{2 1 6 . 4 9 0 . 9 3 8}$ & $2,9 \%$ \\
\hline CANTABRIA & $\mathbf{1 3 6 . 2 8 4 . 8 8 8}$ & $1,8 \%$ \\
\hline CASTILLA Y LEON & $\mathbf{5 6 1 . 9 1 8 . 7 6 5}$ & $7,5 \%$ \\
\hline C. LA MANCHA & $\mathbf{4 1 9 . 7 5 9 . 0 7 5}$ & $5,6 \%$ \\
\hline CATALUÑA & $\mathbf{1 . 3 8 3 . 6 2 0 . 2 3 8}$ & $18,6 \%$ \\
\hline C. VALENCIANA & $\mathbf{4 2 0 . 8 0 5 . 3 8 3}$ & $5,6 \%$ \\
\hline EXTREMADURA & $\mathbf{2 1 2 . 6 1 4 . 0 8 5}$ & $2,9 \%$ \\
\hline GALICIA & $\mathbf{4 4 9 . 9 3 9 . 2 7 6}$ & $6,0 \%$ \\
\hline C. de MADRID & $\mathbf{9 4 4 . 9 0 9 . 5 3 5}$ & $12,7 \%$ \\
\hline MURCIA & $\mathbf{2 1 5 . 1 0 9 . 6 4 6}$ & $2,9 \%$ \\
\hline LA RIOJA & $\mathbf{7 4 . 7 4 4 . 0 5 5}$ & $1,0 \%$ \\
\hline NAVARRA & $\mathbf{7 5 . 0 6 0 . 8 8 3}$ & $1,0 \%$ \\
\hline PAÍS VASCO & $\mathbf{4 6 4 . 3 6 3 . 7 8 3}$ & $6,2 \%$ \\
\hline TOTAL & $\mathbf{7 . 4 4 9 . 6 5 3 . 3 4 0}$ & $\mathbf{1 0 0 , 0 \%}$ \\
\hline
\end{tabular}

Fuente: Imserso. 


\subsection{Financiación por la Administración General del Estado (AGE)}

Todas las aportaciones de la AGE para financiar el coste de atención a la dependencia se realizan a través de transferencias a las comunidades autónomas, ya sea por lo dispuesto en la Ley de dependencia, o en virtud de lo dispuesto en la Ley $22 / 2009$, de I 8 de diciembre, por la que se regula el sistema de financiación de las comunidades autónomas de régimen común y ciudades con estatuto de autonomía y se modifican determinadas normas tributarias, en adelante, Ley de financiación autonómica.

\subsubsection{Financiación estatal regulada en la Ley de dependencia}

La Ley de dependencia en su artículo 7 regula tres diferentes niveles de protección:

I. Nivel mínimo de protección: el establecido por la Administración General del Estado. Se asigna a las comunidades autónomas en función del número de personas beneficiarias con prestación reconocida, su grado de dependencia, así como la tipología de las prestaciones reconocidas 5 .

Existe una estrecha relación entre la gestión llevada a cabo por las comunidades autónomas y los créditos que se transfieren como nivel mínimo: las cantidades que reciben las comunidades autónomas por este concepto dependen, en última instancia, de la cifra de personas beneficiarias con prestación reconocida existentes en cada comunidad, así como de su grado de dependencia reconocido. En consecuencia, un aumento en la cifra de personas beneficiarias con prestación supondrá asimismo un aumento en las cantidades a transferir. En idéntico sentido, el reconocimiento de los mayores grados de

5. Real Decreto I050/2013, de 27 de diciembre, por el que se regula el nivel mínimo de protección establecido en la Ley 39/2006, de I 4 de diciembre, de Promoción de la Autonomía Personal y Atención a las personas en situación de dependencia ( $\mathrm{BOE} \mathrm{n}^{\circ} 3 \mathrm{I} 3$, de 3 I de diciembre de 20I3). dependencia (en especial el Grado III, Gran Dependencia) supondrá asimismo recibir unas mayores cantidades por este concepto, ya que los grados más elevados tienen asociadas unas cifras mayores en concepto de nivel mínimo. Por todo ello, las variaciones en la gestión realizada supondrán asimismo variaciones en las cantidades a transferir como nivel mínimo, de modo que un descenso tanto en la cantidad de personas beneficiarias, como en el reconocimiento de los grados más elevados, supondrá consecuentemente un descenso en la financiación por nivel mínimo.

2. Nivel acordado: el que se acuerde entre la Administración General del Estado y la administración de cada una de las comunidades autónomas a través de los convenios previstos en el artículo ıo de la Ley de dependencia. La disposición transitoria primera de la Ley establece su vigencia únicamente "durante el período comprendido entre el I de enero de 2007 y el 3 I de diciembre de 201 5 , y para favorecer la implantación progresiva del Sistema”, por lo que en la actualidad, 2016 , incluso si no se hubiera suspendido su aplicación, no existiría ya financiación basada en este concepto.

Desde la entrada en vigor de la Ley de dependencia y hasta el año 2015 , último ejercicio completo al redactar estas líneas, la Administración General del Estado, a través del Imserso, ha transferido a las comunidades autónomas ro.955 millones de euros en concepto de nivel mínimo y nivel acordado, como se detalla en la Tabla 5 .

3. Nivel adicional de protección: el que pueda establecer cada comunidad autónoma.

\subsubsection{Financiación estatal regulada en la Ley de financiación autonómica}

Por lo que se refiere a las aportaciones llevadas a cabo en virtud de lo establecido en la Ley de financiación autonómica, desde la entrada en vigor de esta norma legal en 2009, la 
Administración General del Estado, a través del Ministerio de Hacienda, ha transferido a las comunidades autónomas I 5.316 millones de euros en concepto de financiación adicional, tal y como se refleja en el Gráfico 2.

Se puede apreciar, como momento fundamental dentro de la evolución de la financiación del SAAD el ejercicio 2009, año en el que se comenzaron a transferir a las comunidades autónomas los créditos recogidos en la Ley de financiación autonómica.

Mediante esta vía de financiación, como recoge el Tribunal de Cuentas (20I4), se han establecido créditos que, aunque formalmente sin carácter finalista, vienen a sufragar el mayor coste del SAAD para las comunidades autónomas. Además, estas cantidades se calculan precisamente con los mismos criterios con los que se calculaba el nivel acordado, es decir, teniendo en cuenta la población potencialmente dependiente y las personas dependientes con prestación. El Tribunal de Cuentas sostiene primero la procedencia de analizar estos fondos ${ }^{6}$, para después afirmar categóricamente que la financiación adicional no finalista es financiación de la Dependencia ${ }^{7}$ y que tales créditos compensan la suspensión del nivel acordado en 20I2, toda vez que las cifras asociadas a la financiación adicional superan las transferidas en concepto del mencionado nivel acordado ${ }^{8}$.

6. "La referencia a los recursos adicionales, cuya distribución viene exclusiva y directamente vinculada al sistema de dependencia, resulta necesaria para determinar de manera completa la financiación total obtenida por las comunidades autónomas en relación con dicho sistema". Tribunal de Cuentas, Informe de fiscalización I.035/20I4, pág. 62 .

7. "La financiación específica de la dependencia está constituida por el nivel mínimo de protección, a cargo del Imserso, que representó el I9,7 I \% del total. Para el resto de aportación realizada por las comunidades autónomas, éstas recibieron del estado a través de los recursos adicionales no finalistas, si bien directamente vinculados al número de dependientes previstos en la Ley 22/2009, un importe que representó el 29,52\% del total, siendo el $50,77 \%$ restante financiado por las comunidades autónomas a través de otros recursos públicos propios”. Tribunal de Cuentas, Informe de fiscalización I.035/2014, pág. 83 .

8. "Por todo lo anterior, resulta evidente la correlación existente entre los recursos del nivel acordado suspendidos y los recursos adicionales incrementados, atendiendo a la finalidad de ambos, y a su cálculo conforme a unos mismos criterios, sin

\subsubsection{Financiación total de la dependencia por la Administración General del Estado}

De acuerdo con lo expuesto en los dos anteriores apartados, desde la entrada en vigor de la Ley de dependencia en 2007 y hasta 2015 inclusive9, el Estado ha transferido a las comunidades autónomas un total de 26.27 I millones de euros para la financiación de la dependencia, como se aprecia en el Gráfico 4, tanto por la vía finalista como por la adicional.

\section{Gráfico 2. Financiación de la dependencia por la Administración General del Estado 2007-2015 (millones de euros)}

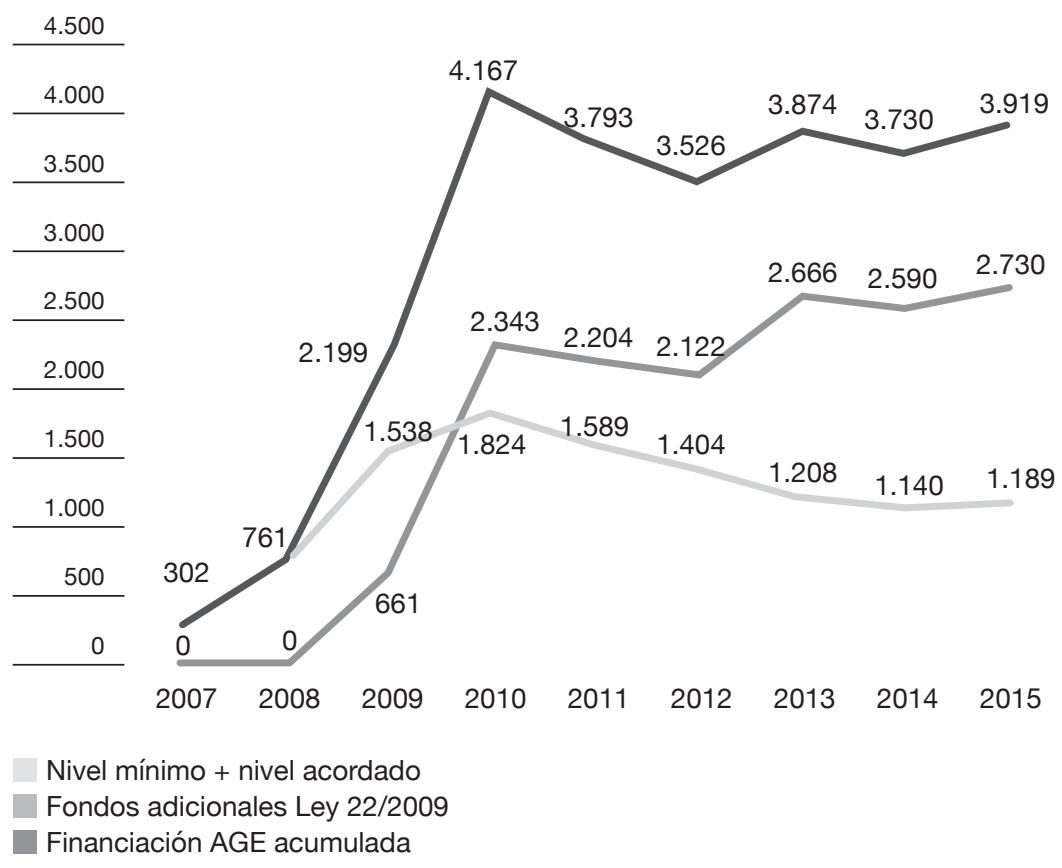

Fuente: Imserso, elaboración propia con base en los datos recogidos en el SISAAD, Presupuestos Generales del Estado y Tribunal de Cuentas.

que ello implique el mismo carácter de financiación afectada”. Tribunal de Cuentas, Informe de fiscalización I.035/20I4. 9. En este cálculo se han tenido en cuenta las cantidades que corresponderían tanto a la Comunidad Autónoma del País Vasco como a la Comunidad Foral de Navarra, a través de la aplicación de los correspondientes porcentajes establecidos en la normativa especial sobre sus regímenes financieros (un 6,24\% de acuerdo con el Concierto Económico entre el Estado y la Comunidad Autónoma de País Vasco, y un I, $60 \%$ de conformidad con el Convenio Económico entre el Estado y la Comunidad Foral de Navarra). 
Durante el periodo correspondiente a este estudio, $20 \mathrm{I} 2$ a 2015 , la financiación estatal del SAAD por vía finalista definida en la Ley de dependencia y por la vía adicional de la Ley de financiación autonómica, ha sido de I 5.049 millones de euros, con el detalle que se muestra en la Tabla 4.

Tabla 4. Financiación estatal del coste del SAAD 2012-2015 (en millones de euros)

\begin{tabular}{|l|l|l|l|l|}
\hline & $\mathbf{2 0 1 2}$ & $\mathbf{2 0 1 3}$ & $\mathbf{2 0 1 4}$ & $\mathbf{2 0 1 5}$ \\
\hline $\begin{array}{l}\text { Fondos finalistas } \\
\text { Ley dependencia }\end{array}$ & 1.404 & 1.208 & 1.140 & 1.189 \\
\hline $\begin{array}{l}\text { Fondos adicionales } \\
\text { Ley financiación } \\
\text { autonómica }\end{array}$ & 2.122 & 2.666 & 2.590 & 2.730 \\
\hline $\begin{array}{l}\text { Total financiación } \\
\text { estatal }\end{array}$ & $\mathbf{3 . 5 2 6}$ & $\mathbf{3 . 8 7 4}$ & $\mathbf{3 . 7 3 0}$ & $\mathbf{3 . 9 1 9}$ \\
\hline
\end{tabular}

Fuente: Imserso, elaboración propia con base en los datos recogidos en el SISAAD, Presupuestos Generales del Estado y Tribunal de Cuentas.

\subsection{Financiación de la dependencia por las comunidades autónomas}

Corresponde a las comunidades autónomas planificar, ordenar, coordinar, dirigir y gestionar, en el ámbito de su territorio, los servicios y recursos necesarios para la atención de la dependencia (artículo i I de la Ley de dependencia).

Además, esta Ley establece en su artículo 32 que "la financiación del sistema será la suficiente para garantizar el cumplimiento de las obligaciones que correspondan a las Administraciones Públicas competentes y se determinará anualmente en los correspondientes Presupuestos" y que "la aportación de la Comunidad Autónoma será, para cada año, al menos igual a la de la Administración General del Estado”. Es decir, de este precepto se deriva la obligación de las comunidades autónomas de aportar cada año una cantidad igual a la recibida por parte de la AGE.
No obstante, no resulta sencillo conocer con exactitud las aportaciones realizadas por las comunidades autónomas para la financiación del SAAD, toda vez que en sus respectivos presupuestos no se recogen de manera específica partidas destinadas a la atención a la dependencia. Tales cantidades suelen venir recogidas en las mismas partidas donde se consignan créditos relativos a otros conceptos de servicios sociales, como la atención a las personas mayores, a las personas con discapacidad o a los servicios sociales básicos. Es más, en el marco de las transferencias que se realizan por parte de las comunidades autónomas a las entidades locales o a entidades sin ánimo de lucro, tampoco resulta posible realizar esta diferenciación.

Esta dificultad ha sido puesta de manifiesto igualmente por el propio Tribunal de Cuentas (20I4) al afirmar que "las comunidades autónomas no han establecido en su ámbito presupuestario, una nítida separación entre los créditos establecidos para la atención de la dependencia y los previstos para los restantes servicios sociales. Asimismo, carecen de sistemas de contabilidad analítica aplicable a los gastos de dependencia y, en su gran mayoría tampoco han dispuesto los instrumentos contables necesarios para efectuar el seguimiento contable de estos gastos, en su consideración de financiación afectada, lo que les impide certificar con exactitud el importe total de los recursos destinados a la dependencia” ${ }^{\text {г. }}$

En tanto que las comunidades autónomas se doten de los "sistemas de contabilidad analítica, que serían necesarios para cuantificar con exactitud el coste de la dependencia, con separación de otros costes de servicios sociales

Io. Al referirse a la aportación de los beneficiarios el Tribunal de Cuentas añade que "los sistemas contables establecidos actualmente tampoco permiten cuantificar con exactitud la aportación de los beneficiarios al coste total de la dependencia". No obstante, sí que aclara el Tribunal que "a la aportación que efectúa la AGE en concepto de nivel mínimo de protección, y en su caso, de nivel acordado, con los correspondientes créditos claramente identificados en los Presupuestos Generales del Estado, no le afectan las dificultades para su determinación que sí afectan a las otras fuentes de financiación”. 
que proporcionan a la ciudadanía” que reclama en su informe, el Tribunal de Cuentas recaba la información disponible y concluye con el reparto de financiación pública entre Estado $(49,23 \%)$ y comunidades autónomas $(50,77 \%)$ que se reproduce en el Gráfico 3, imagen fiel del recogido por el Tribunal de Cuentas en su Informe, en el que se aprecia que, tal y como obliga la Ley de dependencia, la aportación global del Estado y las del conjunto de las administraciones autonómicas, en 20I2, según el Tribunal de Cuentas fueron prácticamente equivalentes.

\section{Gráfico 3. Financiación del SAAD en 2012 según el Tribunal de Cuentas}

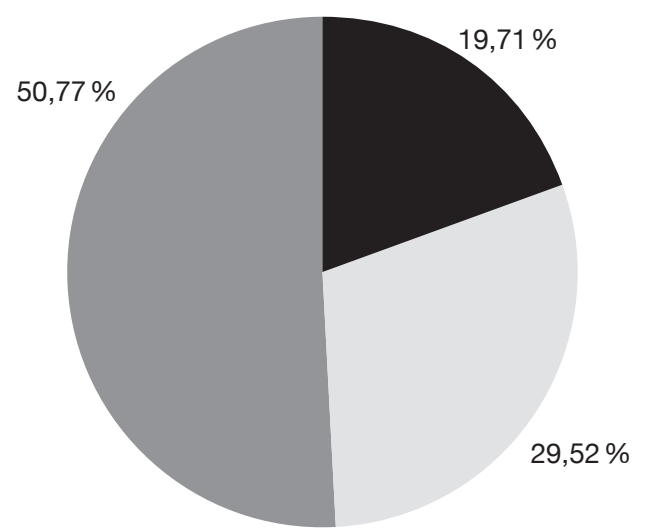

Nivel mínimo Imserso

Recursos adicionales Ley 22/2009

CCAA

Fuente: Tribunal de Cuentas (2OI4). Gráfico II.4-3-I.

Aplicando la lógica del Tribunal de Cuentas, se pueden establecer por diferencia las aportaciones de las comunidades autónomas que, en el periodo 20I2-20I 5 , habrían sido un total de I3.243 millones de euros, de acuerdo con el siguiente desglose anual:

- 20I2:3.369 millones de euros.

- 20I3: 2.984 millones de euros.

- 20I4:3.360 millones de euros.

- 2015:3.530 millones de euros.
Sin embargo, quedaría aún por determinar lo que significan en estas cantidades las aportaciones de los beneficiarios de la Ley de dependencia.

\subsection{Participación de las personas beneficiarias en la financiación del SAAD}

La Ley de dependencia establecía ya en 2006 que, además de las aportaciones de las diferentes administraciones públicas, las personas beneficiarias participen asimismo en la financiación de las prestaciones que reciben ${ }^{\text {II }}$ con un tercio del coste total del SAAD.

Estas previsiones fueron desarrolladas y concretadas por el Acuerdo del Consejo Territorial de de Servicios Sociales y del SAAD para la mejora del Sistema para la Autonomía y Atención a la Dependencia, de ro de julio de $20 \mathrm{I} 2^{12}$, mediante el que se fijaron los criterios y contenidos sobre capacidad económica y participación del beneficiario en el coste de las prestaciones del sistema, cuando proceda. De este modo, se han establecido criterios de procedencia o no de esa participación. Los conceptos que deberán tenerse en cuenta a la hora de determinar la capacidad económica del beneficiario son, principalmente, renta y patrimonio, de los que dependerá la determinación de su participación en la financiación de las prestaciones que recibe. Este hecho puede ayudar a situar en sus justos

I I. El art. 33 de la Ley de dependencia dispone que "los beneficiarios de las prestaciones de dependencia participarán en la financiación de las mismas, según el tipo y coste del servicio y su capacidad económica personal.

2. La capacidad económica del beneficiario se tendrá también en cuenta para la determinación de la cuantía de las prestaciones económicas.

3. El Consejo Territorial del Sistema para la Autonomía y Atención a la Dependencia fijará los criterios para la aplicación de lo previsto en este artículo, que serán desarrollados en los Convenios a que se refiere el artículo io.

Para fijar la participación del beneficiario, se tendrá en cuenta la distinción entre servicios asistenciales y de manutención y hoteleros.

4. Ningún ciudadano quedará fuera de la cobertura del Sistema por no disponer de recursos económicos".

I2. Publicado por Resolución de I 3 de julio de 20 I 2 de la Secretaría de Estado de Servicios Sociales e Igualdad (BOE ${ }^{\circ}$ I 85 , de 3 de agosto). 
términos el debate sobre la participación de los beneficiarios en la financiación del coste del SAAD, ya que esta participación no es de carácter absoluto, sino que tiene límites y su procedencia o no se determina en función de la capacidad económica de la persona.

De un modo similar a lo manifestado a propósito de las aportaciones de las comunidades autónomas, el Tribunal de Cuentas ha señalado en su Informe de fiscalización I.035/20I 4 la dificultad existente a la hora de poder determinar las cantidades aportadas por las personas beneficiarias, al afirmar en su página $8 \mathrm{I}$, que "no resulta posible cuantificar ni siquiera de forma aproximada la participación en la financiación que efectúan a través del copago los propios beneficiarios". En el mismo sentido, Marbán (2015) manifiesta la falta de transparencia de las comunidades autónomas en la contabilización de la aportación de los beneficiarios, lo que añade dificultad a la realización de comparativas fiables.

En todo caso, la nueva normativa sobre el nivel mínimo recogida en el citado Real Decreto I050/2013, de 27 de diciembre, ha incorporado la obligación de las comunidades autónomas de aportar anualmente un certificado donde se recoja el gasto realizado en la materia. De este modo, a partir de las mencionadas certificaciones se podrá conocer con una mayor precisión las cantidades aportadas por las comunidades autónomas. En esta misma línea, el mencionado Real Decreto y la nueva Orden reguladora del SISAAD establecen la obligación para las comunidades autónomas de incluir la aportación de los beneficiarios como dato obligatorio en el Sistema, de tal manera que en el futuro se podrá conocer esta información.

Con el fin de contar con información al respecto, en el Imserso se ha realizado un ejercicio de cálculo de estas aportaciones, estimando la capacidad económica de las personas beneficiarias con base en los datos económicos de las mismas (tramos de rentas medias, pensiones, etc.) y aplicando los criterios de determinación de la aportación de la persona beneficiaria para cada servicio o prestación, en función de su capacidad económica, contenidos en el Acuerdo del Consejo Territorial de Servicios Sociales y del SAAD de 2012 antes referido. La estimación de las aportaciones de los beneficiarios, obtenida para el periodo objeto 2OI 2-20I 5, ha sido de 7.774 millones de euros, de acuerdo con el siguiente desglose por años:

- 2012: r.848 millones de euros. Un $26,8 \%$ del total.

- 20I3: r.947 millones de euros. Un $28,4 \%$ del total.

- 20I4: r.900 millones de euros. Un $26,8 \%$ del total.

- 2015: 2.079 millones de euros. Un $27,9 \%$ del total.

En unas cifras bastante similares se encuentra el estudio realizado por Prada y Borge (20I4) en el que, como se aprecia en el Gráfico 4, para el ejercicio 2013 estimaron una cantidad de 1.870 millones de euros en concepto de aportación de los beneficiarios, un $28,73 \%$ del total. Para ello, siguieron una metodología similar a la utilizada en el Imserso, teniendo en cuenta diferentes tramos de renta de las personas beneficiarias.

\section{Gráfico 4. Aportación de los beneficiarios al coste total de la dependencia en 2013, según Prada y Borge}

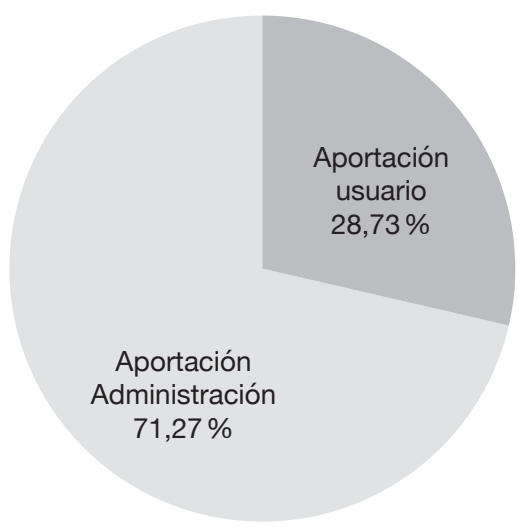

Fuente: Prada y Borge, 20I4. 
3.4. Financiación global de la dependencia: aportaciones de la Administración General del Estado, de las comunidades autónomas y de los beneficiarios

Llegados a este punto se pueden presentar ya los cálculos de la financiación del coste de la dependencia realizados. En la Tabla 5 se muestran los resultados, teniendo en cuenta los tres sumandos anteriormente expuestos:

- La aportación de la Administración General del Estado: tanto la financiación finalista del nivel mínimo de protección estatal derivada de la Ley de dependencia, como la financiación adicional no finalista derivada de la Ley de Financiación de las Comunidades Autónomas de Régimen Común, así como el cálculo correspondiente a las Comunidades de Régimen Especial (País Vasco y Navarra).

- La aportación de recursos propios de las comunidades autónomas: estimación obtenida por diferencia con las aportaciones del Estado y de los beneficiarios.

- La aportación de los beneficiarios: estimada a partir de su capacidad económica.

\begin{tabular}{|c|c|c|c|c|}
\hline & 2012 & 2013 & 2014 & 2015 \\
\hline $\begin{array}{l}\text { Administración } \\
\text { General del Estado }\end{array}$ & 3.526 & 3.874 & 3.730 & 3.919 \\
\hline $\begin{array}{l}\text { Comunidades } \\
\text { autónomas } \\
\text { recursos propios }\end{array}$ & 1.521 & 1.037 & 1.464 & 1.451 \\
\hline Beneficiarios & 1.848 & 1.947 & 1.896 & 2.079 \\
\hline $\begin{array}{l}\text { TOTAL } \\
\text { FINANCIACION } \\
\text { COSTE } \\
\text { DEPENDENCIA }\end{array}$ & 6.895 & 6.858 & 7.090 & 7.449 \\
\hline
\end{tabular}

Fuente: Imserso. Elaboración propia.

En la Tabla 5 resulta apreciable cómo las aportaciones realizadas por cada una de las tres partes, durante los años 20 I 2 a 2015 en los que nos detenemos en este estudio, fueron distintas a las previstas por la Ley de dependencia y su Memoria de Impacto:

- La aportación de la Administración General del Estado a la financiación de la atención a la dependencia supuso más del tercio del coste total del Sistema previsto en la Memoria de Impacto de la Ley de dependencia. Para el ejercicio 2012 las aportaciones de la AGE supusieron el 5 I,I \% del coste total, en el año 2013 un $56,5 \%$ e igual porcentaje para los años $20 \mathrm{I} 4$ y $20 \mathrm{I} 5$, un 52,6I \%.

- Las estimaciones de las aportaciones de recursos propios de las comunidades autónomas estuvieron por debajo de la financiación del Estado y de las previsiones de la Memoria de Impacto de la Ley de dependencia: un 22, I \% en 20I2, un I $5, \mathrm{I} \%$ en $20 \mathrm{I} 3$, un $20,64 \%$ en $20 \mathrm{I} 4 \mathrm{y}$ un I $9,5 \%$ en 2015.

- La estimación de las aportaciones de los mismos beneficiarios se acercan a las previsiones iniciales de la Ley de dependencia y de su Memoria: un 26,8\% en $20 \mathrm{I} 2,28,4 \%$ en $20 \mathrm{I} 3,26,75 \%$ en $20 \mathrm{I} 4$ y $27,9 \%$ en 2015 .

Finalmente, el estudio de estas diversas vías de financiación puede realizarse para cada una de las comunidades autónomas y por cada uno de los años de 2012 a 2015 . En la Tabla 6 de este trabajo se recoge la distribución de las aportaciones para la financiación del coste del SAAD por comunidades autónomas, tan solo para el último ejercicio objeto de estudio en este artículo por razones de espacio y dado que los valores tanto absolutos como relativos son muy estables a lo largo del periodo estudiado ya que, como vimos al principio de este trabajo, la cifra de beneficiarios atendidos por el SAADa de $20 I 2$ a 20 I 4 se ha mantenido bastante estable en torno a las 750.000 personas y en el año 2015 , coincidiendo con la implantación total de la Ley de dependencia, los beneficiarios con prestación han aumentado hasta los 796.Io9. 

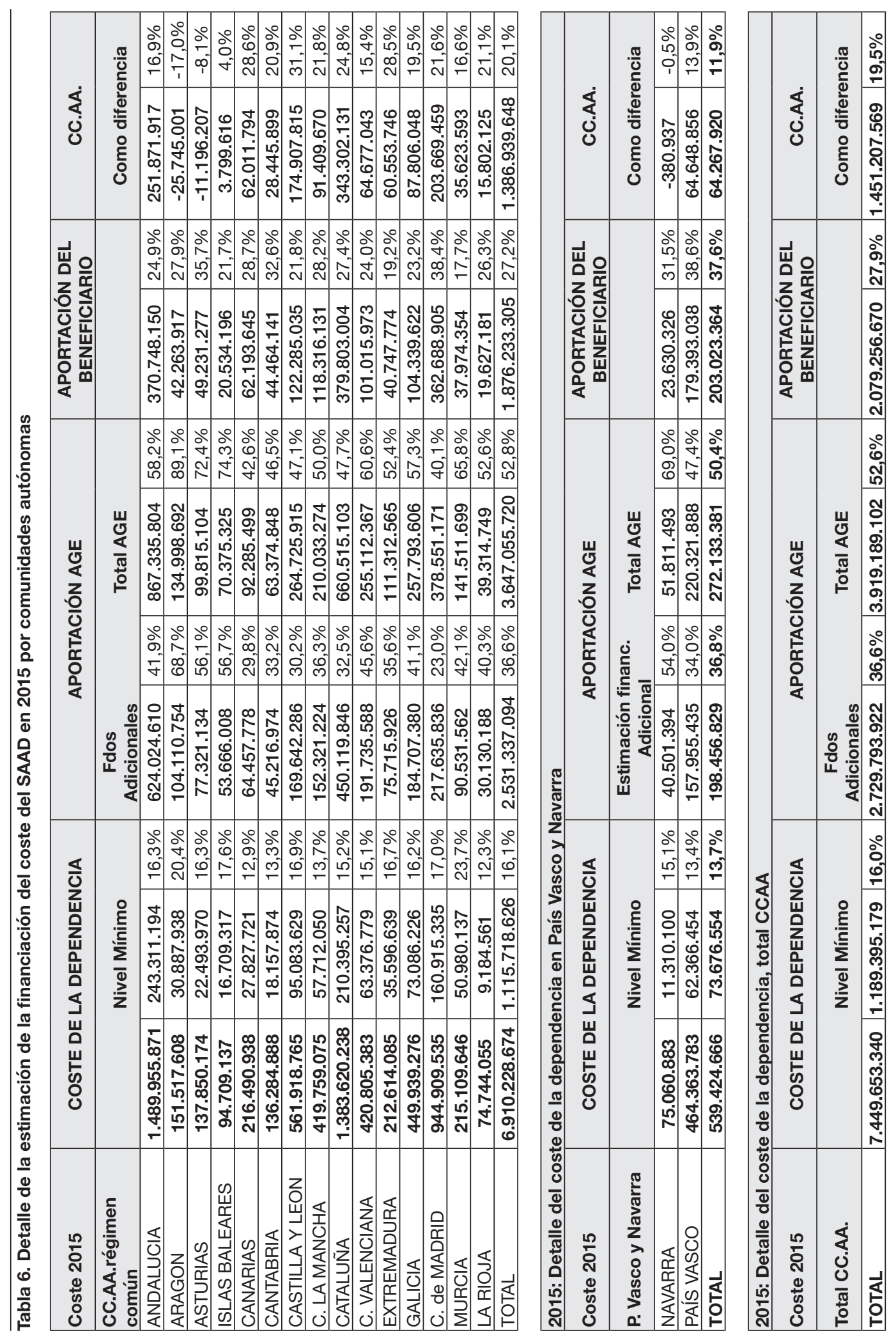

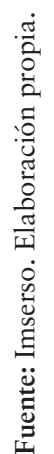


El Tribunal de Cuentas (20I4) ya puso de relieve que, por parte de la AGE, se estaba cumpliendo el mandato legalmente establecido de financiar, al menos, la mitad del coste del SAAD.

En el Gráfico 5 se recoge la representación gráfica que realiza el Tribunal, en el Informe de fiscalización citado, de los porcentajes reales de las aportaciones autonómicas, en las comunidades de régimen común, a la financiación de la dependencia certificadas, esto es muy relevante, por las propias administraciones autonómicas. Es decir, el Tribunal de Cuentas afirma, con información de las propias comunidades autónomas, que éstas -junto con los beneficiarios- se encuentran aportando entre el $73 \%$ de la financiación total del sistema (Madrid) y el $27 \%$ (Galicia). autónomas al Tribunal de Cuentas, durante el año 2012 las aportaciones de la AGE alcanzaron una media del 59,23\% del coste total, mientras que las aportaciones de las CCAA (incluidas las aportaciones de los beneficiarios), supusieron el $50,77 \%$.

Y por último, debe asimismo reseñarse que el estudio de Prada y Borge (2014) llega a unas conclusiones similares, puesto que en el mismo se recoge que para el ejercicio $20 \mathrm{I}_{3}$ las aportaciones efectuadas por la AGE alcanzaron el 54,77\% del coste total de la atención a la dependencia, mientras que las aportaciones de los presupuestos autonómicos supondrían tan sólo el I 6,50\%, y las aportaciones de los beneficiarios serían de un $28,73 \%$, como se puede apreciar en el Gráfico 6.

De este modo, con base en la información facilitada por las propias comunidades

\section{Gráfico 5. Financiación de la dependencia por CCAA, según el Tribunal de Cuentas}

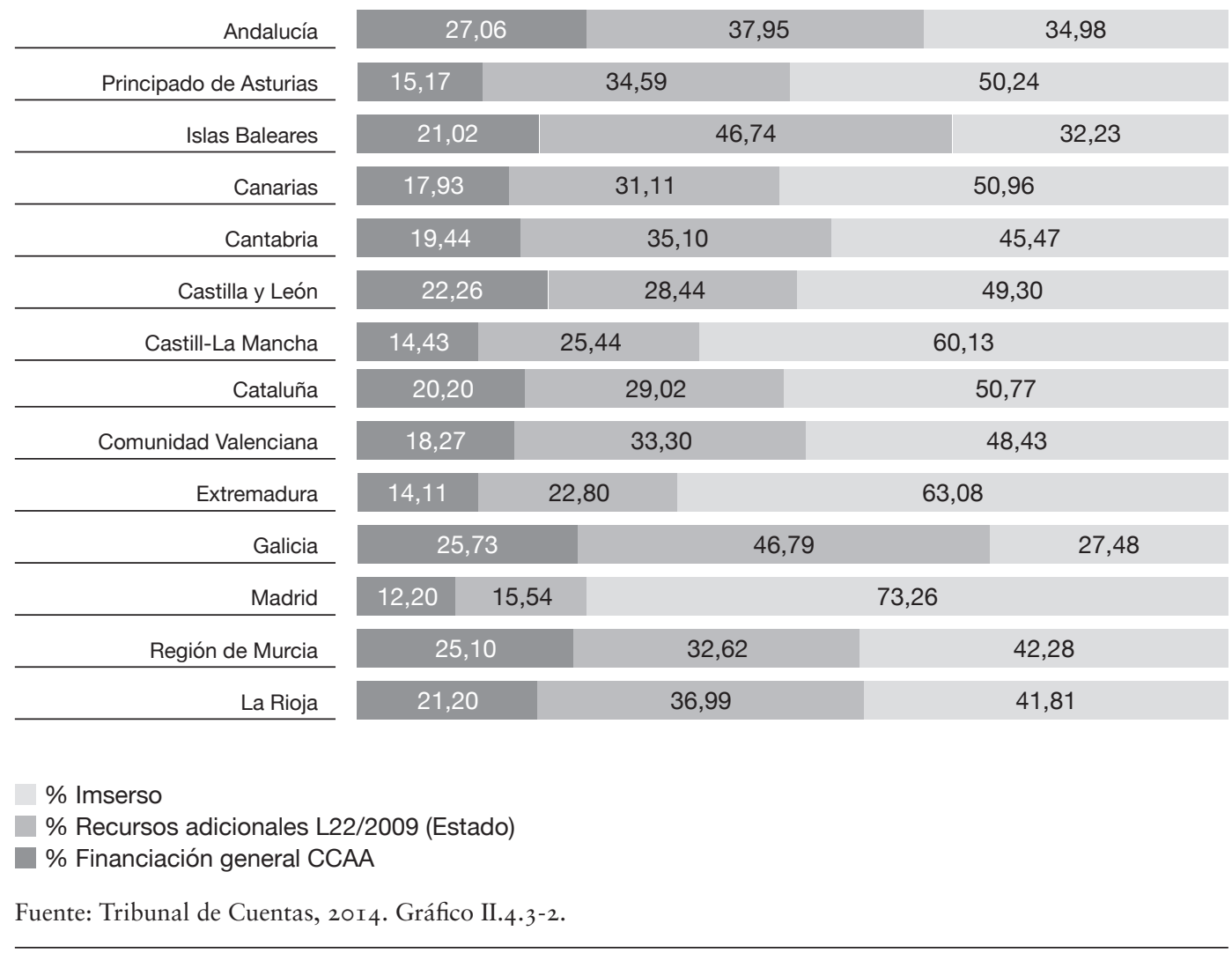




\section{Gráfico 6. Financiación de la dependencia según Prada y Borge}

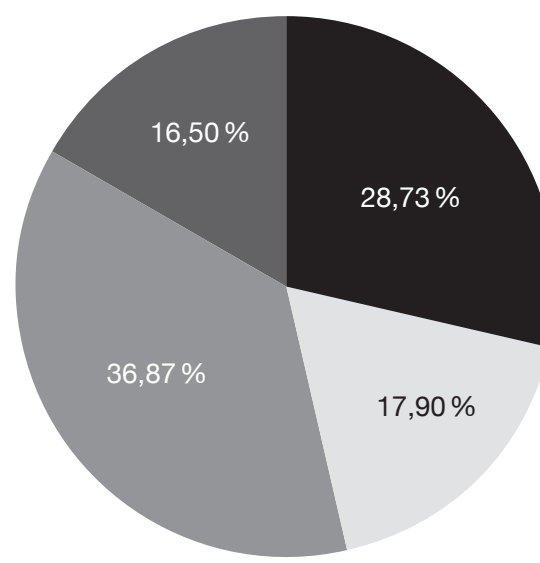

Beneficiarios

Nivel mínimo

Financiación adicional

CCAA

Fuente: Prada y Borge, 20I4.

\section{Conclusiones}

Como resumen y conclusiones de este estudio podemos enumerar las siguientes:

I. El coste del SAAD en cada uno de los años del periodo 20I2-20I 5 habría sido:

- 20I2: 6.895 millones de euros.

- 2013: 6.858 millones de euros.

- 20I4: 7.090 millones de euros.

- 20I5: 7.449 millones de euros.

2. La financiación de este coste se realiza por el Estado, las comunidades autónomas y los propios beneficiarios.

3. La financiación del Estado se rige por dos vías distintas: finalista y adicional o no finalista, reguladas por sendas leyes que garantizan las aportaciones a realizar por parte del Estado a las comunidades autónomas para financiar el coste de atención a la dependencia:

- $\quad$ La Ley de dependencia, la financiación finalista, a través del nivel mínimo de protección y, en su caso, del nivel acordado.

- La Ley de financiación autonómica, de carácter no finalista, que viene a sufragar el mayor coste del SAAD para las comunidades autónomas y que se calcula, precisamente, con los mismos criterios con los que se calculaba el nivel acordado, es decir, teniendo en cuenta la población potencialmente dependiente y las personas dependientes con prestación.

4. Durante todo el período de vigencia de la Ley de dependencia, 2007-20I 5, la Administración General del Estado ha destinado a las comunidades autónomas 26.27 I millones de euros, tanto por la vía finalista como por la adicional o no finalista.

5. En el periodo 2OI 2-20I 5, objeto de este estudio, la Administración General del Estado ha destinado a las comunidades autónomas I 5.049 millones de euros, tanto por la vía finalista como por la no finalista.

6. En concreto, en 20 I $_{5}$, último ejercicio completo del que se disponen cifras, se han transferido desde el Estado a las comunidades autónomas 3.919 millones de euros. Esta cantidad supone el 52,6I \% del coste anual del SAAD. El resto habría sido financiado por comunidades autónomas $($ I $9,48 \%)$ y beneficiarios $(27,9$ I \% ). En el resto de años del periodo 20I 2-20I 5 esta distribución relativa ha resultado muy similar. 
Referencias bibliográficas

Asociación Estatal de Directoras y Gerentes de Servicios Sociales (2012): Demolición controlada de la Ley de Dependencia (en línea). <http://www.directoressociales. com/2-uncategorised/I64-denuncian-unademolici $\% \mathrm{C}_{3} \% \mathrm{~B}_{3}$ n-controlada-de-la-ley-dedependencia.html>, acceso 6 de diciembre de 2016.

Beitia, R. (20I 5 ): "La sostenibilidad del Sistema para la Autonomía y Atención a la Dependencia durante el trienio 2OI2-20I4: el equilibrio entre costes y financiación". Zerbitzuan: Gizarte zerbitzuetarako aldizkaria, Revista de servicios sociales, 60: 3 I-46.

España. Real Decreto I050/2013, de 27 de diciembre, por el que se regula el nivel mínimo de protección establecido en la Ley 39/2006, de I 4 de diciembre, de Promoción de la Autonomía Personal y Atención a las personas en situación de dependencia, Boletín Oficial del Estado, 3I de diciembre de 20I3, núm. 3 I3, pp. IO7I 20.

España. Resolución de I 3 de julio de 20I2, de la Secretaría de Estado de Servicios Sociales e Igualdad, por la que se publica el Acuerdo del Consejo Territorial del Sistema para la Autonomía y Atención a la Dependencia para la mejora del sistema para la autonomía y atención a la dependencia, Boletín Oficial del Estado, 3 de agosto de 20I2, núm. I85, pp. 55657 .

España. Real Decreto-ley 20/20I2, de I3 de julio, de medidas para garantizar la estabilidad presupuestaria y de fomento de la competitividad, Boletín Oficial del Estado, I4 de julio de 20I2, núm. I68, pp. 50428.

España. Ley 22/2009, de I 8 de diciembre, por la que se regula el sistema de financiación de las Comunidades Autónomas de régimen común y Ciudades con Estatuto de Autonomía y se modifican determinadas normas tributarias, Boletín Oficial del Estado, I9 de diciembre de 2009, núm. 305, pp. 107087.
España. Ley 39/2006, de I4 de diciembre, de Promoción de la Autonomía Personal y Atención a las personas en situación de dependencia, Boletín Oficial del Estado, I 5 de diciembre de 2006, núm. 299, pp. 44I42.

Imserso (20I6): Avance de la evaluación 2015 del Sistema para la Autonomía y Atención a la Dependencia (en línea). <http://www. dependencia.imserso.es/InterPresentı/groups/ imserso/documents/binario/im_I02607.pdf>, acceso 6 de diciembre de 2016 .

Imserso (2015): Avance de la Evaluación anual del ejercicio 2014 de los resultados de la aplicación de la Ley de Dependencia (en línea). <http://www.dependencia.imserso. es/InterPresent I/groups/imserso/documents/ binario/estsisaad20 I 4 I 23 I av.pdf $>$, acceso 6 de diciembre de 2016 .

Imserso (201 5): Servicios Sociales dirigidos a personas mayores en España. Diciembre de 2012 (en línea). <http://www.imserso.es/ InterPresentr/groups/imserso/documents/ binario/recursos_sociales_20I 2.pdf>, acceso 6 de diciembre de 2016.

Imserso (20I4): Evaluación anual ejercicio 2013 de los resultados de aplicación de la Ley de Dependencia (en línea). <http://www. dependencia.imserso.es/dependencia_or/ evo_doc/e/eva_20I3/index.htm>, acceso $6 \mathrm{de}$ diciembre de 2016 .

Imserso (20I3): Evaluación anual ejercicio 2012 de los resultados de aplicación de la Ley de Dependencia (en línea). <http://www. dependencia.imserso.es/InterPresentı/groups/ imserso/documents/binario/evaluacion_ ley_20I2.pdf $>$, acceso 6 de diciembre de 2016 .

Imserso (20I2): Evaluación de resultados a I de enero de $20 \mathrm{I} 2$ de la Ley de Dependencia (en línea). <http://www.dependencia.imserso. es/InterPresent2/groups/imserso/documents/ binario/ield_20I2.pdf>, acceso 6 de diciembre de 2016. 
Marbán Gallego, V. (20 I 5 ): “La gobernanza del sistema y las diferencias interterritoriales en el SAAD". Documentación social, I77: 4I-60.

Ministerio de Trabajo y Asuntos Sociales (2006): Memoria del análisis de impacto normativo, económico, de género e igualdad de oportunidades, no discriminación y accesibilidad universal. Anteproyecto de Ley de promoción de la autonomía personal y de atención a las personas en situación de dependencia (en línea). <https://www. inforesidencias.com/resources/public/biblioteca/ documentos/ley-de-dependencia/memoria-leydependencia.pdf $>$, acceso 6 de diciembre de 2016.

Montserrat, J. (20I3): "La ley de dependencia en caída libre". Agathos: Atención sociosanitaria y bienestar, 2: 62-64.

Prada, M. D. y Borge, L. M. (201 5 ): “Una aproximación al coste de la dependencia en
España y su financiación”. Documentación social, I77: 97-II8.

Tribunal de Cuentas (20I4): Informe de fiscalización sobre las medidas de gestión y control adoptadas por las Comunidades Autónomas para la adecuada aplicación de la Ley 39/2006, de I4 de diciembre, de Promoción de la Autonomía Personal y Atención a la Dependencia (en línea). <http:// www.tcu.es/repositorio/ec 5 afa2e-d9e6-4 I Ic825 I-I75636a40726/II035.pdf >, acceso $6 \mathrm{de}$ diciembre de 2016 .

Tribunal de Cuentas (2013): Informe de fiscalización de la gestión económico-financiera $y$ de la aplicación de la Ley 39/2006, de I4 de diciembre, de Promoción de la Autonomía Personal y Atención a la Dependencia (en línea). <http://www.congreso.es/docu/inf fiscTC/25I-24.pdf $>$, acceso 6 de diciembre de 2016. 